\title{
Monte carlo study of the percolation in two-dimensional polymer systems
}

\author{
Monika Pawlowska • Andrzej Sikorski
}

Received: 13 October 2012 / Accepted: 14 May 2013 / Published online: 14 June 2013

(C) The Author(s) 2013. This article is published with open access at Springerlink.com

\begin{abstract}
The structure of a two-dimensional film formed by adsorbed polymer chains was studied by means of Monte Carlo simulations. The polymer chains were represented by linear sequences of lattice beads and positions of these beads were restricted to vertices of a two-dimensional square lattice. Two different Monte Carlo methods were employed to determine the properties of the model system. The first was the random sequential adsorption (RSA) and the second one was based on Monte Carlo simulations with a Verdier-Stockmayer sampling algorithm. The methodology concerning the determination of the percolation thresholds for an infinite chain system was discussed. The influence of the chain length on both thresholds was presented and discussed. It was shown that the RSA method gave considerably lower thresholds for longer chains. This behavior can be explained by a different pool of chain conformations used in the calculations in both methods under consideration.
\end{abstract}

Keywords Lattice models · Monte Carlo method .

Percolation · Polymers

\section{Introduction}

Percolation is a phenomenon in which a cluster formed by given objects becomes infinite, i.e., in real systems it extends from one border of the system to another [1]. In general, one of the common theoretical procedures is to determine the percolation threshold, i.e., the minimum concentration of objects at which the percolations too place. It has all features of the phase transition and thus, from many years it has been an object of theoretical interest [1]. The

M. Pawłowska $\cdot$ A. Sikorski $(\bowtie)$

Department of Chemistry, University of Warsaw, Pasteura 1, 02-093 Warsaw, Poland

e-mail: sikorski@chem.uw.edu.pl percolation theory has a lot of applications in physics, especially in disordered systems and porous media. Computer simulation of this process can usually be realized by random sequential adsorption (RSA) [2-4]. RSA can model the adsorption of atoms and small molecules, binding of large ligands to polymer chains or protein to solid surfaces, polymers or biological membranes. The lattice approximation is commonly used for the consideration of the percolation in many systems in order to suppress the degree of freedom and for computational purposes. The objects or their parts (beads) are located in vertices of a quasicrystalline lattice - in such a case a cluster is formed when an object has neighbors, i.e., the distance between objects (or parts of objects) is equal to the lattice unit. Usually, the percolation problem is classified as site percolation where sites are blocked and bond percolation where bonds joining the sites are blocked. One has to remember that the percolation of the polymer chain is a special case of bond-site percolation where bonds are correlated because the chains are integral objects.

The most popular large objects studied using the RSA method were stiff elongated rods (needles) [5-10]. The percolation in such a system was found to be quite different from that for other large objects: the percolation threshold does not change monotonically with the length of the object because needles tend to form locally ordered clusters. In spite of rather extensive studies there are still some inconsistencies in the description of the scaling behavior of percolation and jamming thresholds with aspect ratios of these objects. Systems containing other elongated objects (ellipsoids, rectangles) were also studied and it was shown that the aspect ratio was a crucial parameter that determined the percolation threshold [11-13].

The consideration of the percolation phenomenon in systems containing other large and irregular objects like polymer chains requires some studies to evaluate calculation methods for this purpose. Short flexible chains (oligomers) were studied 
up to date mainly by means of the RSA method by [14-18]. Pandey et al. studied the percolation and jamming (the maximum coverage) in such systems but it is difficult to refer to their results because of quite different determination of the thresholds $[14,15]$. Nieto et al. performed a detailed and a solid study in which they concentrated on the universality of the percolation process and the influence of the lattice representation on it $[16,17]$. Recent studies of two-dimensional polymer systems performed by Zia et al. have shown interesting behavior of the bond percolation threshold [19-22], however, this model was different from others as polymer chains can cross themselves. The percolation of semi-flexible chains was recently studied by the RSA method [23] showing the temperature dependencies of percolation threshold and a non monotonic behavior of these threshold at some conditions. Mixtures of small objects (oligomers) of a different shape were also studied by means of the RSA method [24] while mixtures of stiff rods and flexible chains appeared to be tractable by a theory (a connectedness Ornstein-Zernike formalism) [25]. Long flexible chains were recently studied by means of the RSA [10] and the CMA (the cooperative motion algorithm) [26-29] simulation methods. The influence of the presence of explicit solvent molecules, the analysis of the clusters formed by macromolecules and the confirmation of the universal critical behavior of these systems were the main results discussed in these works.

In this paper we studied the structure of two-dimensional systems consisting of flexible linear polymer chains near the percolation threshold. The representation of polymer chains was very simple: macromolecules were represented by the sequences of identical beads and positions of these beads were restricted to vertices of a square lattice. The excluded volume was the only polymer-polymer potential. The main question addressed was to compare and evaluate methods used for studies of the percolation in systems containing long flexible chains. The paper is organized in the following way: in section The model and the algorithms we describe in detail the idealized representation of polymer chains and both simulation methods used (RSA and a Metropolis-like algorithm). In section Results and discussion we present the results, mainly percolation thresholds and discuss the comparison of simulation methods and their evaluation. The section Conclusions contains the most important conclusions concerning comparison of results and validating of both simulation methods.

\section{The model and the algorithms}

The polymer representation

Flexible linear polymer chains were approximated by sequences of beads without any atomic details and single bead represented some chemical mers. Each chain in the system consisted of $N$ beads and the number of chains was $n$. In order to make the calculations more efficient a lattice approximation was introduced: the positions of polymer beads were limited to vertices of a square lattice. The excluded volume was the only potential introduced into the model and, therefore, the chains could not cross themselves. No local potential was introduced and thus, the chains were fully flexible. Other long-distance interactions were assumed to be identical, i.e., the system was athermal and corresponded to good solvent conditions. The system was studied in a large two-dimensional Monte Carlo box and the edge of this box $L$ was chosen to be large enough when compared to the chain's diameter in order to avoid the chain interacting with its image and in order to minimize the influence of the system's size on the percolation threshold. Periodic boundary conditions were also introduced in both directions. The problem concerning the finite size of the Monte Carlo box in our studies will be discussed below in the next section. The structure of the polymer system was determined by means of two different simulation methods.

The Metropolis-like algorithm

The properties of the systems under consideration were calculated by means of the Monte Carlo simulation employing an algorithm based on local changes of chain's conformation (Fig. 1a). The set of these local moves consisted of: 1-bead motion, 2-bead motion, 1-bead end modification and 2-bead end modifications [30]. Additionally we used wave motions, where fragments of chain (three consecutive segments) were transferred randomly along the chain and exchanged with a single bond and the reptation motion. These last two micromodifications are essential for the ergodicity of the process and make the relaxation of the system considerably faster [30-32]. A polymer bead was picked randomly and an attempt of a motion was performed and accepted/rejected due to excluded volume and geometrical constraints. A Monte Carlo cycle was defined as one attempt of each local motion applied in average to one bead. An initial polymer's conformation was constructed in a process of the simultaneous growing and the equilibration procedure: a system containing $n$ chains each containing $N$ beads was eventually built. For each system under consideration 10-15 independent Monte Carlo simulation runs were performed. Each simulation run consisted of $10^{7}-10^{10}$ cycles and at the start of each simulation run the equilibration run was performed, which lasted $10^{6}$ $10^{9}$ cycles. The criterion of the equilibration of the system was the stability of some time-mean parameters of the system, such as the end-to-end distance and the radius of gyration. 
a

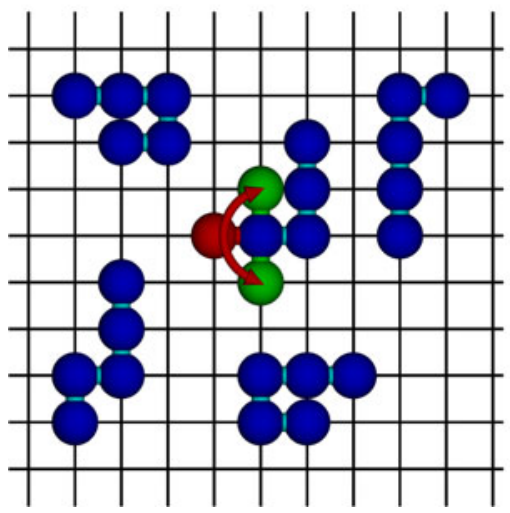

Fig. 1 Schemes of algorithms used in simulations. All two-dimensional chains are present on the surface (in blue) and they undergo local changes of conformation (old conformation is marked in red and the new ones in

\section{Random sequential adsorption}

In this method polymer chains were subsequently adsorbed on a substrate. We considered a Monte Carlo box $L \times L$ for this purpose. A two-dimensional chain was randomly selected from an earlier prepared library of conformations and put on the substrate. The adsorbed macromolecules could not overlap and this was realized by the forbidding of the double occupancy of lattice points by polymer beads. All chains were immobilized, e.g., no conformational changes were allowed and the diffusion process was forbidden (Fig. 1b). Simulation started with an empty substrate and for each chain at first we randomly selected the position of the chain's beginning. Then, we checked all sites along the chains contour. If all these sites were empty, the new chain was added to the system, otherwise the entire procedure was repeated. A chain adsorbed on the substrate formed a new cluster. If any element of the added chain was at the distance of one lattice unit from an already deposited chain (or a cluster of chains) the newly added chain formed one cluster with them.

Before the RSA process we prepared libraries containing a collection of polymers' conformations for a given chain length $N$. For shorter chains the libraries were filled with all possible conformations. For longer chains the libraries were created using the modifications of chain conformations by the algorithm described in section The polymer representation. Libraries contained up to $10^{7}$ different conformations and these conformations were independent one from the other, i.e., each of the chains was recorded after a large time step. We used the libraries to avoid creating non-overlapping polymer chains during the simulations (and problem with biased statistics when generating self-avoiding walks) and thus to speed up the RSA process significantly. When neighboring chains formed continuous path from one system edge to the opposite, this was called a percolation. In order to recognize

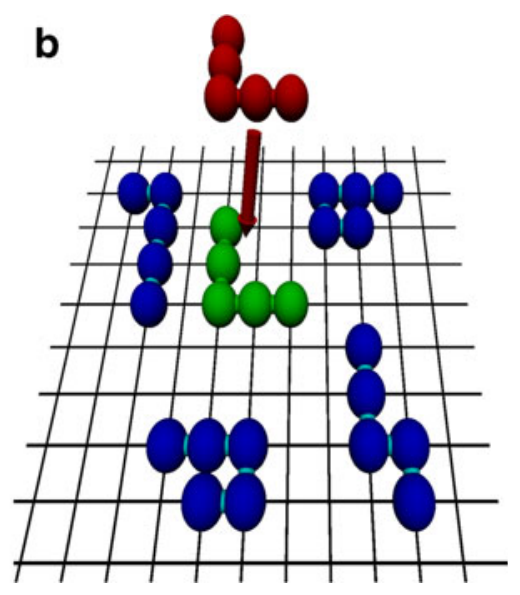

green) (a). Random sequential adsorption where a two-dimensional chain (in red) is put on a surface and accepted (in green) when it doesn't crosses with chains already present on the surfaces (in blue) (b)

the moment of percolation a Hoshen \& Kopelman-like algorithm was applied. In this cluster identification algorithm one travels over all lattice points row-wise and identifies clusters consisting of neighboring beads. The tree of labels indicates the connections between the identified clusters [1, 33]. The RSA procedure was repeated for each system 100 1000 times.

\section{Results and discussion}

Simulations were performed for a chain length $N$ from 3 to 500 (the Metropolis-like algorithm) and for $N$ from 1 to 500 (the RSA method). In order to determine the influence of the size of the system on the percolation threshold the simulations were performed in the Monte Carlo box with the edge $L=50,100,200,1000$ and 2000 . The polymer concentration was defined as a fraction of lattice sites occupied by chains, i.e., the number of polymer beads to the total number of sites in the system: $\varphi=n N / L^{2}$.

The structure of the adsorbed polymer layer was frequently a subject of theoretical and simulation studies [34]. Therefore, we will focus on the percolation in chain systems only. Using the Metropolis-like algorithm one has to detect if a given configuration of the system contains a percolated polymer cluster or not. Therefore, we can determine the percolation probability calculated as the ratio of the number of percolated systems to the total number of configurations generated for a given set of parameters. Figure 2 presents the percolation probability $P$ as a function of the polymer concentration $\varphi$. All $P(\varphi)$ curves are typical, i.e., S-shaped and the increase of the chain length shifts the curve toward lower polymer concentrations. Qualitatively the same behavior was found for other two-dimensional polymer chains: for short chains on the square lattice [17], long linear chains with explicit solvent molecules on the triangular lattice [26] 


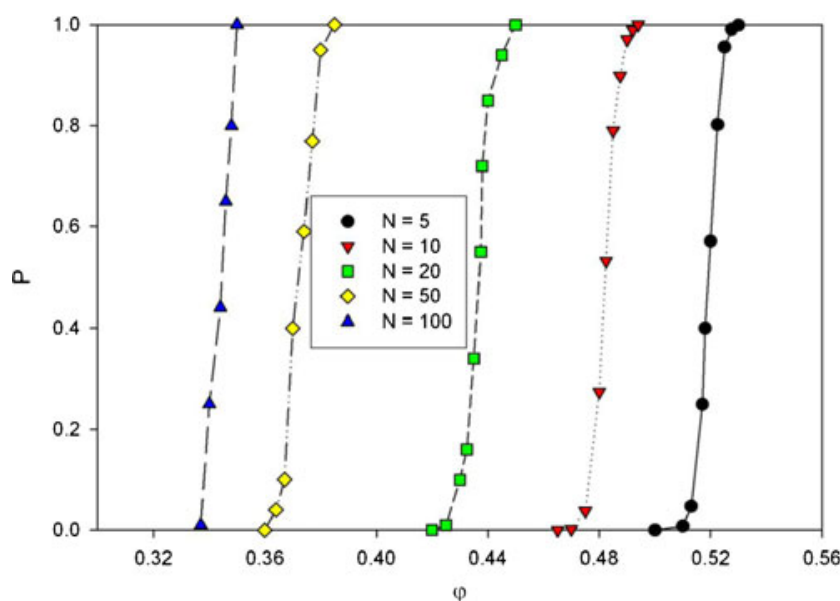

Fig. 2 Plot of the percolation probability $P$ obtained in the MC method as a function of the polymer concentration $\varphi$. The case of the Monte Carlo box $L=1000$. Chain lengths are given in the inset

and the square lattice [21] and for off-lattice hard ellipsoids [11]. One can also observe that the slope of curves decreases with the chains length which is caused by the finite size of the system.

The percolation threshold can be determined from the Sshaped probability curves as an inflection point. In order to calculate the value of the thresholds for infinitely large system two procedures were proposed. The first one finds the inflection point on a $P(\varphi)$ curve using the following fitting [17, 35]:

$P(\varphi)=1-\left(1+\exp \left(\frac{\varphi-c_{p}}{a}\right)\right)^{-1}$

where $a$ is a constant that determines the slope of the curve and with $a \rightarrow 0$ the curve reduces to the step function (for an infinitely long chain). The next step involved the finite-size scaling analysis. The extrapolation of the percolation threshold to the thermodynamic limit was done by fitting to the scaling relation:

$\left|c_{p}(L)-c_{p}(\infty)\right|^{\sim} L^{-1 / \nu}$,

where $c_{p}(L)$ and $c_{p}(\infty)$ are percolation thresholds for the Monte Carlo box $L \times L$ and for an infinite system respectively, and $\nu$ is a critical exponent (theoretical predictions give $\nu=4 / 3$ ) [1]. In Fig. 3 we show the calculated values of $c_{p}$ versus $(L / N)^{-1 / n}$. All curves are linear, i.e., the extrapolation of the percolation thresholds toward an infinite system can be made according to Eq. (2). One can also notice from this figure that the corrections to the thresholds obtained from simulations in a large MC box $(2000 \times 2000)$ are on the order of $10^{-3}$, which is below the standard error.

The second method of the determination of the percolation threshold for an infinite system is based on the finding

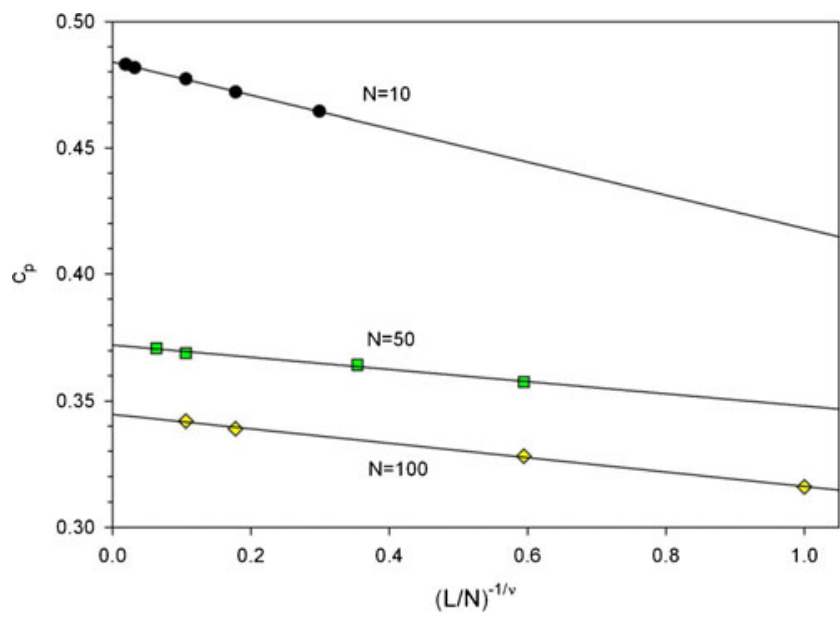

Fig. 3 Log-log plot of the percolation thresholds $c_{p}$ obtained in the $\mathrm{MC}$ method as a function of the $(L / N)^{-1 / n}$ for some chain lengths

that percolation probability curves $P(\varphi)$ for systems of different size intersect in one point $[11,21,36]$. Figure 4 shows the percolation probabilities $P$ as functions of the polymer concentrations $\varphi$ for the chain $N=10$ calculated for some sizes of the Monte Carlo box $L$. The increase of the system makes the probability curve steeper (for the infinite system one expects the step function). All curves intersect in one point which can give the value of the percolation threshold for the infinitely large box. The values obtained using this methodology are exactly the same as those obtained in the first method. Both series of the percolation thresholds are presented in Table 1.

The percolation thresholds calculated via both simulation techniques are presented in Fig. 5 and plotted against the chain length $N$. One can observe that the threshold decreases with increase of the length of the macromolecule (the number of beads comprising the chain) regardless of the simulation method used. There is one more interesting observation

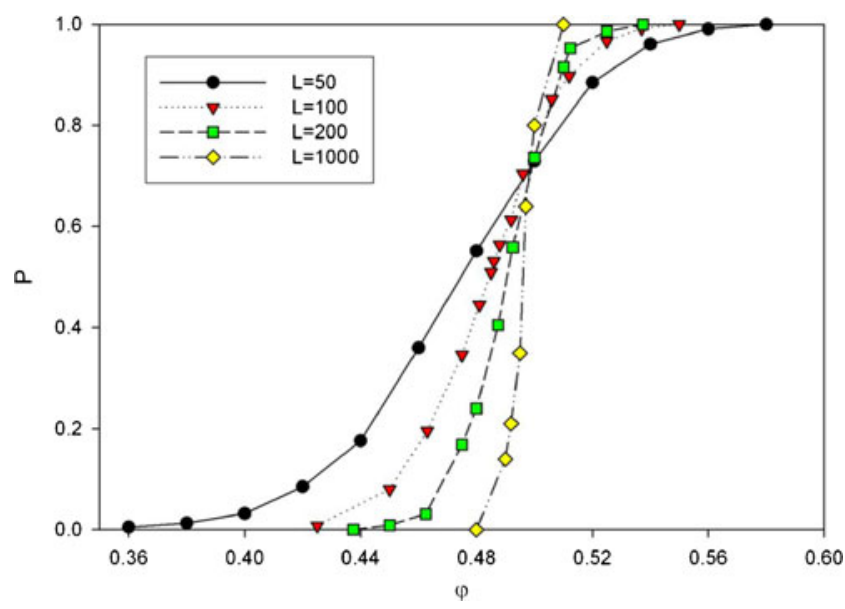

Fig. 4 Plot of the percolation probability $P$ obtained via the MC method as a function of the polymer concentration $\varphi$. The case of chain $N=10$. The sizes of the Monte Carlo box $L$ are given in the inset 

tion thresholds obtained by the RSA and MC methods. The values of thresholds are extrapolated toward infinite system (see text for details).
Table 1 The percola-

\begin{tabular}{lll}
\hline $\mathrm{N}$ & $\mathrm{RSA}$ & $\mathrm{MC}$ \\
\hline 1 & $0.592(2)$ & - \\
2 & $0.562(2)$ & - \\
3 & $0.549(2)$ & $0.550(2)$ \\
5 & $0.523(2)$ & $0.521(3)$ \\
10 & $0.480(3)$ & $0.484(3)$ \\
20 & $0.428(5)$ & $0.439(4)$ \\
30 & $0.398(5)$ & $0.411(4)$ \\
40 & $0.375(8)$ & $0.387(6)$ \\
50 & $0.354(6)$ & $0.375(8)$ \\
75 & $0.325(8)$ & $0.354(10)$ \\
100 & $0.315(11)$ & $0.345(9)$ \\
200 & $0.282(12)$ & $0.320(11)$ \\
300 & $0.261(14)$ & $0.305(12)$ \\
300 & $0.244(15)$ & $0.291(11)$ \\
400 & $0.230(17)$ & $0.278(14)$ \\
\hline
\end{tabular}

concerning the behavior of the percolation thresholds: the values obtained in the MC method are always higher than those from the RSA method and this difference increases with the chain length (at least for the chain lengths studied). The reason of the difference between RSA and MC predictions will be discussed below.

The question arises what would be the percolation threshold for infinitely long flexible chains? To answer this question one should consider the polymer local density, i.e., the number of polymer segments within an equivalent ellipse defined as $<N / S^{2}>$. For a single chain $<S^{2}>$ increases faster than the number of segments because $<S^{2}>$ scales as $N^{3 / 2}$ for two-dimensional systems. Therefore, the local density approaches zero for chains of the infinite length. The percolation threshold corresponds to the system near the crossover from dilute to semidilute solution $[37,38]$. The chains that are separated and behave mainly independently start to

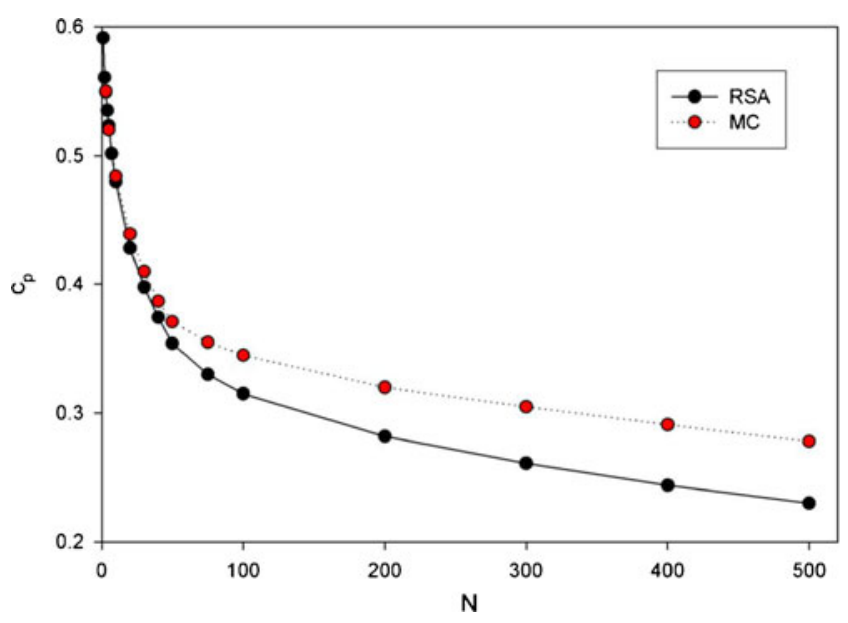

Fig. 5 Plot of percolation thresholds $c_{p}$ for as a function of chain length $N$ obtained via both methods studied touch each other. Thus, the polymer density in the entire system near this crossover should approach zero as within a single chain. On the other hand, the number of chains in an infinite Monte Carlo box would also be infinite. Therefore, it is difficult to state if the percolation threshold, which decreases with the length of chain that was observed in our simulations, will eventually approach zero or other non-zero value.

A formula describing the influence of the chain length on the percolation threshold was proposed by Nieto et al. [16]. It was based on the Galam and Maugier equation formulated for a site and bond percolation:

$c_{p}=p_{o}[(d-1)(c-1)]^{-a}$,

where $d$ is the dimension of the system, $c$ is lattice coordination number while $p_{o}$ and $a$ are fitting parameters. Nieto and al. extended this formula for chains consisting of $N$ elements giving the following expressions for both parameters $a$ and $p_{o}$ :

$a(N)=A_{a} \exp \left(-N / B_{a}\right), \quad p_{o}(N)=A_{p_{o}} \exp \left(-N / B_{p_{o}}\right)$.

$A_{a}, B_{a}, A_{p o}$ and $B_{p o}$ are new fitting parameters. It has to be stressed that in this formula $c_{p}$ approaches zero when the chain length becomes infinite. Unfortunately, the fitting parameters A and B have no physical meaning. On the other hand, the next publication of Nieto at al. [17] suggests nonzero value of the percolation threshold for infinitely long chain. In order to describe the dependency of the percolation threshold on the chain length they proposed a different formula:

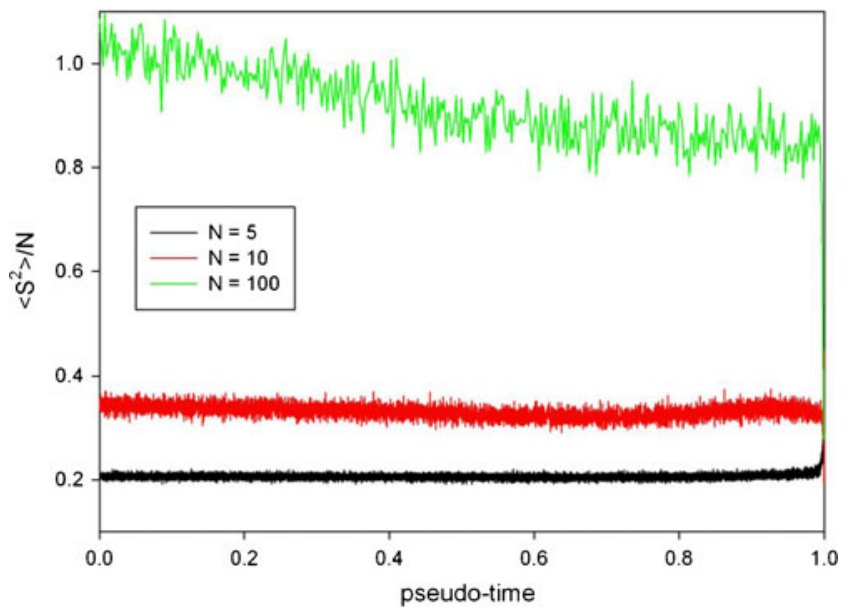

Fig. 6 The reduced mean-squared radius of gyration $<S^{2}>/ N$ of a chain successfully put on the substrate as a function of the pseudo-time in the RSA method (see text for details). The chain lengths are given in the inset 


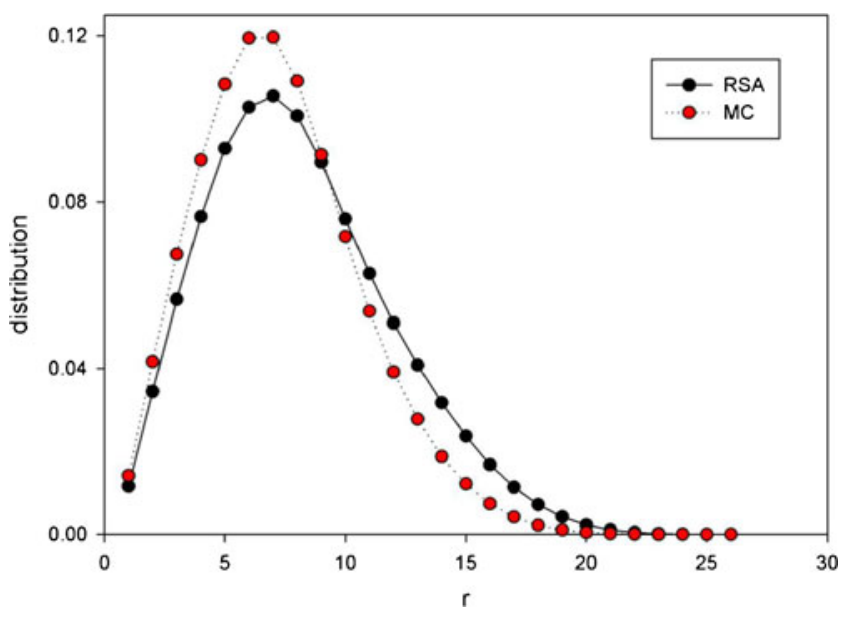

Fig. 7 The distribution of polymer beads around the chain's center of mass for the chain $N=80$ obtained via both methods studied

$c_{p}=c_{p}^{*}+\Omega \exp \left(-\frac{\kappa}{N}\right)$

where $c_{p}{ }^{*}, \Omega$ and $\kappa$ are fitting parameters and the parameter $c_{p}{ }^{*}$ corresponds to the percolation threshold for the infinitely long chain. The fitting of their data gave $c_{p}{ }^{*}=0.461 \pm$ 0.001 but it has to be stressed that Nieto et al. studied very short chains only $(N \leq 15)$. Fitting of our data gives a similar result for short chains $\left(c_{p}{ }^{*}=0.447\right)$ while quite a different one for the entire range of the chain lengths $\left(c_{p}{ }^{*}=0.261\right)$.
Thus, the parameters of Eq. (5) depend on the chain length and the formula is not valid.

One has to remember that the percolation threshold depends on the chain's fractal dimension, which is characteristic for the entire class of objects. This hypothesis was already validated for a slightly different model of twodimensional linear and cyclic chains on triangular lattice with explicit solvent molecules introduced [26, 27]. This suggests that the main factor, which determines the percolation threshold of a complex object, is its structure.

It was shown that the RSA method gave lower percolation threshold when compared to those obtained by MC simulations. This discrepancy can be explained in the following way. The size of a single chain can be expressed by means of the radius of gyration $<S^{2}>$, i.e., the mean-squared distance between polymer beads belonged to the same chains and its center of mass. In the MC method all chains have size that is characteristic for a given polymer density and in average each chain has the same dimension. In the RSA method the situation is different. Figure 6 presents the values of the chains' reduced mean-squared radius of gyration $<S^{2}>/ N$ as a function of the pseudo-time. Pseudo-time is a number of a chain put successfully on the substrate divided by the number of chains that had been approached after reaching the percolation threshold. These reduced values are necessary in order to study size's changes for chains of different length. For short $(N=5$ and 10) chains their size along with the RSA procedure
Fig. 8 Snapshots of the system at the percolation threshold for systems consisted of chains $N=5$ (a), $N=10$ (b), $N=50$ (c) and $N=100$ (d)
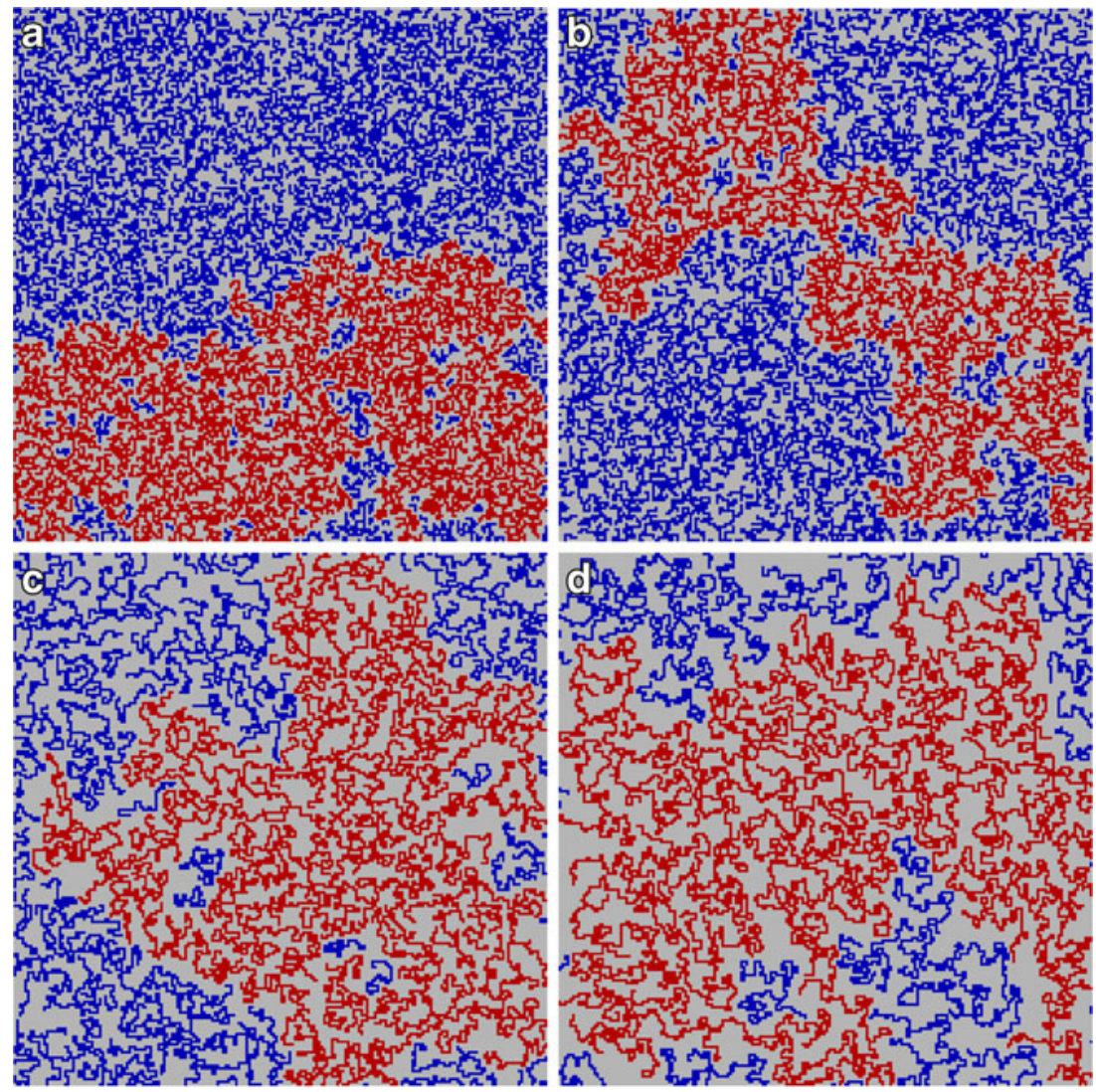
almost does not change - for these chains the dependency of the polymer size on the density of the system is rather small. The size of long chains $(N=100)$ considerably decreases during the adsorption process. It should be expected as each next chain meets smaller space to be fit in. This behavior was implicitly confirmed by Wang and Pandey who had found that the radius of gyration of deposited chains (in the RSA method) was less sensitive to the polymer density and that chains featured memory of time of deposition [15]. The differences in the average structure of a single chain in both methods used can be studied by the analysis of the spatial distribution of polymer beads in a chain. Figure 7 presents the distributions of polymer beads around the chain's center of mass. One can observe that the shape of both curves is similar while the distribution of beads of the chain used in the MC method is slightly broader. This distribution of segments confirms that the only difference between average chains in both methods is their size.

It is possible to obtain the same percolation threshold in both methods. For this purpose one has to prepare a different library of chains conformation from which chains are randomly picked up and put on the substrate. This library should contain conformation typical for a polymer solution at the density near the percolation threshold determined via the RSA method. Then, the next library containing conformations typical for the density equal to the new percolation threshold should be prepared and the next RSA procedure should be performed. This iterative procedure should give the same percolation threshold as the MC method.

The differences in the structure of the surface covered by a film formed of short and long flexible linear macromolecules can also be visualized. Figure 8 presents typical snapshots of some polymer systems under consideration taken at the percolation threshold. The decreasing density of the system is clearly visible as the percolation threshold changes here from 0.521 to 0.345 . The percolation clusters are the largest among other clusters and their size increases when the chain length increases. The number of polymer beads in percolation clusters considerably decreases when going from short to long chains. One can also observe the presence of non-percolated islands inside the percolation cluster and the decreasing number of these non-percolating islands when going from short to long chains.

\section{Conclusions}

An idealized polymer model was developed in order to study the percolation in systems containing long linear flexible macromolecules. In this model the polymer chains were represented as sequences of identical beads and the positions of these beads were restricted to vertices of a square lattice. The model systems were athermal, i.e., the excluded volume was the only potential introduced. Two different computer simulations techniques were used to determine the properties of the polymer systems: random sequential adsorption and Monte Carlo simulations with a Metropolis-like algorithm.

It was shown that the usage of the random sequential adsorption method led to lower percolation thresholds in the entire range of the chain lengths studied. The percolation threshold decreases in the entire range of lengths studied while for shorter chains $(N \leq 50)$ this decrease is rather rapid. Because of these differences in prediction of the percolation threshold, the question of validity of the RSA method for long flexible chain systems arises. One can argue that the RSA method simulates the systems where the macromolecules precipitate from a diluted solution at good solvent conditions and are firmly bound by the surface (the adsorption is irreversible, for instance because of chemical bonds). On the other hand, in the Metropolis method adsorbed objects can move along the surface and their conformations are typical for polymer systems near the crossover between the diluted and semidiluted solution. In other words, the average size of a polymer chain in the Metropolis method is smaller than that of an isolated chain used in the RSA method which enables better packing and results in lower percolation thresholds.

Acknowledgments The computational part of this work was done using the computer cluster at the Computing Center of the Department of Chemistry at the University of Warsaw. This work was partially supported by the Polish Ministry of Science and Higher Education grant N N204 118539.

Open Access This article is distributed under the terms of the Creative Commons Attribution License which permits any use, distribution, and reproduction in any medium, provided the original author(s) and the source are credited.

\section{References}

1. Stauffer D, Aharony A (1994) Introduction to percolation theory. Taylor and Francis, London

2. Evans JW (1993) Rev Mod Phys 65:1281-1329

3. Talbot J, Tarjus G, Van Tassel PR, Viot P (2000) Colloid Surface A $165: 287-324$

4. Wang JS (2000) Colloid Surface A 165:325-343

5. Vigil RD, Ziff RM (1989) J Chem Phys 91:2599-2602

6. Ziff RM, Vigil RD (1990) J Phys A: Math Gen 23:5103-5108

7. Vandewalle N, Galam S, Kramer M (2000) Eu Phys J B 14:407-410

8. Kondrat G, Pekalski A (2001) Phys Rev E 63:051108

9. Kondrat G, Pekalski A (2001) Phys Rev E 64:056118

10. Adamczyk P, Romiszowski P, Sikorski A (2008) J Chem Phys 128:154911

11. Garboczi EJ, Snyder KA, Douglas JF, Thorpe MF (1995) Phys Rev E 52:819-828

12. Yi YB, Sastry AM (2004) Proc Royal Soc Lond A 460:2353-2380

13. Vygornitskii NV, Lisetskii LN, Lebovka NI (2007) Colloid J 69:557-562

14. Becklehimer JL, Pandey RB (1994) J Stat Phys 75:765-771

15. Wang SJ, Pandey RB (1996) Phys Rev Lett 77:1773-1776 
16. Cornette V, Ramirez-Pastor AJ, Nieto F (2003) Physica A 327:7175

17. Cornette V, Ramirez-Pastor AJ, Nieto F (2003) Eur Phys J B 36:391-399

18. Sung BJ, Yethiraj A (2010) Phys Rev E 81:031801

19. Gopalakrishnan M, Schmittmann B, Zia RKP (2004) J Phys A: Math Gen 37:L337-L343

20. Schmittmann B, Gopalakrishnan M, Zia RKP (2005) J Phys: Condens Matter 17:S1817-S1825

21. Wu Y, Schmittmann B, Zia RKP (2008) J Phys A: Math Theor 41:025004

22. Zia RKP, Wu Y, Schmittmann B (2009) J Math Chem 45:58-64

23. Kondrat G (2002) J Chem Phys 117:6662-6666

24. Lončarević I (2007) Budinski - Petković L, Vrhovac SB. Eur Phys J E 24:19-26

25. Wang X, Chatterjee AP (2003) J Chem Phys 118:10787-10793

26. Adamczyk P, Polanowski P, Sikorski A (2009) J Chem Phys $131: 234901$

27. Żerko S, Polanowski P, Sikorski A (2012) Soft Matter 8:973-979
28. Pawłowska M, Żerko S, Sikorski A (2012) J Chem Phys 136:046101

29. Sikorski A, Polanowski P, Adamczyk P, Żerko S (2011) J Mol Model 17:2209-2215

30. Kolinski A, Vieth M, Sikorski A (1991) Acta Phys. Polon A 79:601-612

31. Skolnick J, Kolinski A (1990) Adv Chem Phys 77:223-278

32. Binder K, Müller M, Baschnagel J (2004) Polymer Models on the Lattice. In: Kotelyanskii M, Theodorou DN (eds) Simulation methods for polymers. Dekker, NewYork

33. Hoshen J, Kopelman R (1976) Phys Rev B 14:3438-3445

34. Eisenriegler E (1993) Polymers Near Surfaces. World Scientific, Singapore

35. Tarasevich YY, Cherkasova VA (2007) Eur Phys J B 60:97-100

36. Saven JG, Skinner JL, Wright JR (1991) J Chem Phys 94:6153-6159

37. des Cloizeaux J, Jannink G (1990) Polymers in solution. Their modelling and structure. Clarendon Press, Oxford

38. Teraoka I (2002) Polymer solutions. An introduction to physical properties. Wiley, New York 